\title{
Distributed Data Transaction of an Apache Web Server using Bulk Service Rule
}

\author{
V.B.Kirubanand \\ Assistant Professor \\ Computer Application Department \\ SKCET College, Coimbatore- 8
}

\author{
Dr.S.Palaniammal \\ Professor and Head \\ Science and Humanities Department \\ VLBJCET College, Coimbatore- 42.
}

\begin{abstract}
The main theme of this paper is to find the Distributed Data Transaction of an Apache web server using bulk service rule. We obtain the parameter of service rate, Arrival rate, Expected waiting time and Expected Busy period. The inter arrival and inter service of HTTP request is assumed to Poisson Distribution Process (PDP) and these events are considered in the server for process sharing. The total number of requests are processed, there is no time limited to arrivals. While compared to some models, our model of $\mathrm{M} / \mathrm{M}(1, \mathrm{~b}) / 1$ is more efficient to find response and request time in between client and server. This model has been validated through java programming. The performance has been found in the model of $\mathrm{M} / \mathrm{M}(1, \mathrm{~b}) / 1$ which fits well to the practical outcome in client and web server.
\end{abstract}

Keywords: World Wide Web, Web Server, bulk service rule, PDP,

\section{INTRODUCTION}

Client-Server System are becoming increasingly common in the world today as users move to networks of distributed, interacting computer through internet. This process of work demands new performance modeling in the interaction in client server systems and are more complex solvers in parameters like inter arrival and service time, Root of specification, Excepted waiting time and Expected busy period.

In this paper we consider the Apache web server described in [3] and [2] which is a well-known web server. This facilitates the integration of both hardware and software aspects of the system behavior in the improved model. This lends itself very well to modeling in distributed component-based system [3] such as modern e-business applications.

In this world million of people hit index per day and there will be traffic in inter arrival rate and inter service rate in between client and web server. The overload of data packets arrival means there will be traffic in between client and web server and some of the data packets are rejected.[4].

A number of attempts occur to create performance model of web server [5]. The model which is used to predict the web server performance modeling validates through measurements and simulation [6] made a performance evaluation of web server using wired network. This model has several parameters, which are known. The unknown parameters are determined by simulation [7] used in a layered queuing model.

The user gets much benefit in sharing data packets through wired devices (switch) in internet access to find the performance modeling in between the client and server using markovian models of bulk service rule.
Some of the models are congested in the data packets in arrival as well as service period. The performance modeling of an apache web server with busy arrival traffic [8] using $\mathrm{M} / \mathrm{M} / 1 / \mathrm{K}$ found in a (FCFS) services description can predict web server performance quite well.

A very simple model like the $\mathrm{M} / \mathrm{M} /(1, \mathrm{~b}) / 1$ using queuing models, will predict the inter arrival and inter service in the web server and client. The performance of that model is quite possible to work.

In this paper we have derived a model of the Apache web server [1] which includes a processor sharing node to one system to another. The inter arrival and service process in between web server and clients is assumed with the help of PDP. MMPP are commonly used to represent busy arrival traffic to communication system [9], but we use PDP to find the average arrival rate and service rate. Both are considered in the distribution process. The average arrival rate and service rule is assumed with bulk systems.

The average service time and the maximum number of jobs are parameters that can be determined through a minimum likelihood estimation,[10]. After completing the Poisson distribution process we had found the root of specification in between the web server and clients. By simulating the system, we are able to obtain the web server and client performance measures such as request and response time probability. We prefer the validation environments provided in a server and client connected through a switch, the evaluation validates the parameter in the model. The solution shown in the model can predict the performance measures in both lighter data packet and overloaded data packets.

\section{WEB ERVER AND CLIENT MODEL}

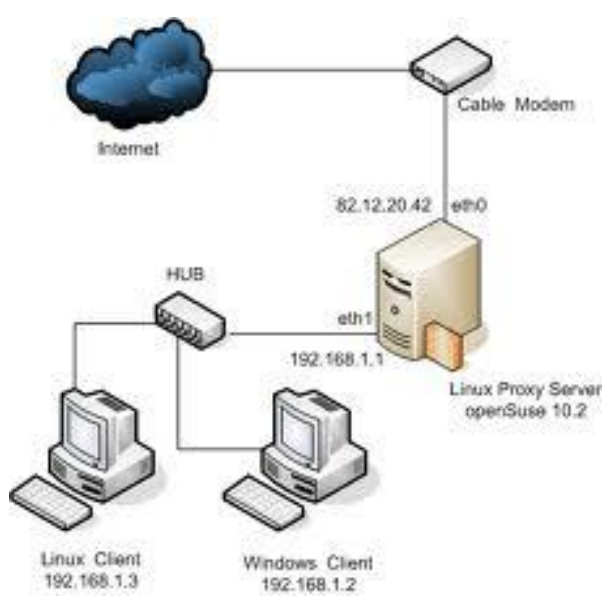

Figure 1. Client server model connected with number of clients with web server 
Switch can be used for wired networking like the popular router, it is a second generation protocol that allows higher data rates over larger distances, efficient use of bandwidth, and avoids interference almost at minimum [11].

We model the web server and clients using markovian model in bulk service rule of $\mathrm{M} / \mathrm{M}(1, \mathrm{~b}) / 1$. $\mathrm{M} / \mathrm{M}(1, \mathrm{~b}) / 1$ clients get much benefit when compared to $\mathrm{M} / \mathrm{M} / 1 / \mathrm{K}$.

While using $\mathrm{M} / \mathrm{M}(1, \mathrm{~b}) / 1$ bulk service rule, client requests are taken to server for service in a bulk(more than one), not one by one. A waiting time in queue is abridged, and clients don't need to wait for long in a queue to get services. Similarly a model of an M/M/1 bulk queue with service rate dependent on the batch size is developed. [12]

The service can handle at the most ' $\mathrm{K}$ ' request at a time. A request will be blocked if the number ' $\mathrm{K}$ ' has been reached. [13] .The $\lambda$ is the rate of completed request and response $\mu$ i.e average response of time ' $T$ ' probability are performance measures which will be provided in the simulation using java programming.

\section{EXPERIMENTAL RESULTS}

1. PDP Parameter

We calculate using $\lambda / \mu$, to find the average response and request time.

2. Root of specification

The threshold value of Root of specification lies between $0<\mathrm{r}>1$

3. Inter arrival time

It is the time taken between client and server (i.e request)

4. Inter service time

It is the time taken between server and client (i.e response)

5. Expected waiting time density

$$
E(T)=\frac{r}{\left.\mu\left(1-r^{b}\right)+r\left(1-r^{b}\right)\right]}
$$

6. Expected Busy Period

$$
E(B)=\frac{1}{\mu(1-r)}
$$

\section{ENCRYPTION TECHNIQUE \\ 4.1 Cryptography}

In cryptography the structure of the message is scrambled to make it meaningless and intelligible unless the decryption key is available. I make no attempt to disguise or hide the encoded message basically; cryptography offers the ability of transmitting information between client and server in the way that prevents from third party hacking. Cryptography can also provide authentication for verifying the identity of someone or something

\section{PERFORMANCE MEASURES}

We consider the validation measurements used single server computer and multi client computer which are connected through $100 \mathrm{mbps}$. The server is Intel ${ }^{\circledR}$ core Duo Processor, 2.0 GHZ, 2MB LZ cache memory, 1GB DDR2 RAM, 160GB Serial ATA 7200RPM Hard Disk.
The computer representing the client is a processor of 2.99GHZ, RAM- 1GB. Both server and client computers were connected through windows XP and windows server 2003 operating system. Apache web server is also installed in the server virtually. We use different hardware configuration in between client and server. Maximum users access the data from server to client.'

We proceed with the following performance measures to the average of request and response time, root of specification, and inter arrival and service time, expected waiting time and busy period. The request time is the time difference in response time. i.e. the data request to server and the server response data to the client. The average response and request time is calculated using Poisson distribution process (PDP). After measuring the request and response time it is forwarded to the root of specification. This will be provided to the expected waiting time and busy period. Similarly, with the measures of root of specification, we can include these values to the waiting time and busy periods measurements.

The model of $\mathrm{M} / \mathrm{M} / 1 / \mathrm{K}$ and $\mathrm{M} / \mathrm{M}(1, \mathrm{~b}) / 1$ are client and server through switch devices. [13].The results of the performance modeling is done in the java program. An HTTP request is sent by a client computer to the server computer. A TCP/IP connection is timed out at the client computer (request) when it will take a long time to the server to return (acknowledge).[14].The number of systems ' $\mathrm{K}$ ' is the model, we use the some parameter that were found. [15]. Where a similar model was used with PDP arrivals instead of MMPP arrivals. The same parameter, where it is not new parameter has been obtained in the models [16]. This model will be a correct solution measure. We can use this parameter for future devices

\section{SIMULATION MODEL}

We considered performance modeling in wired device like SWITCH Data transition. We perform the simulation for Data Transaction in between Client and Apache Web Server.

TABLE 1. Network Parameter Specification

\begin{tabular}{|l|l|}
\hline Mean Arrival Rate Per Node & 300 Packets/Sec \\
\hline Mean Service Time & $5 \mathrm{Sec}$ \\
\hline Threshold number of Packets & 1 to 20 \\
\hline Root of Specification & $0<\mathrm{r}>1$ \\
\hline
\end{tabular}

Simulation results are obtained for various scenarios by varying the number of nodes and threshold number of packets per node in a network data transaction. Simulation results clearly show that there exists trade-offs between the server and data service. And also the results show that the average of number of packets.

\section{CALCULATION PARAMETERS}

$\lambda$ - Inter arrival time in between client and web server

$\mu$ - inter service time in between client and web server

$\mathrm{b}$ - Batch size of bulk arrival

$\mathrm{R}-$ Root of Specification $0<\mathrm{r}>1$

$E(t)$ - expected waiting time of client from service of server

E (b) - expected busy period of server

\section{RESULTS AND DISCUSSION}

In this experiment we measure the performance modeling using bulk service rule by simulation with java program. The values are measured in the client and server with different configurations and also operating system. The value with corresponding measurement shows the average response and 
request time. Irrespective of number of request each configurations are having constant threshold for root of specification value measured in request and response time rate in $\mathrm{M} / \mathrm{M} /(1, \mathrm{~b}) / 1$ model. The request time and response time is calculated in different configuration using $\mathrm{M} / \mathrm{M} /(1, \mathrm{~b}) / 1$. This way of finding (Lam) and (meu) is given result when compared to $\mathrm{M} / \mathrm{M} / 1 / \mathrm{K}$ model.

The Simulation and Analytical results obtained are presented in graph model. By assuming the Data transaction in between Client and Web server.

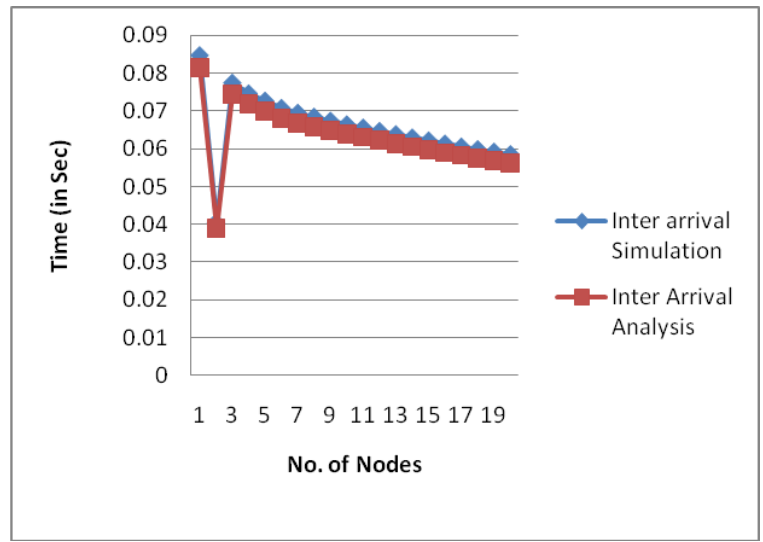

Figure 1. Simulation and Analysis of Inter Arrival time.

Its shows that number of nodes in between 1 and 20 . In $X$ Axis and Times in sec provided in Y Axis.

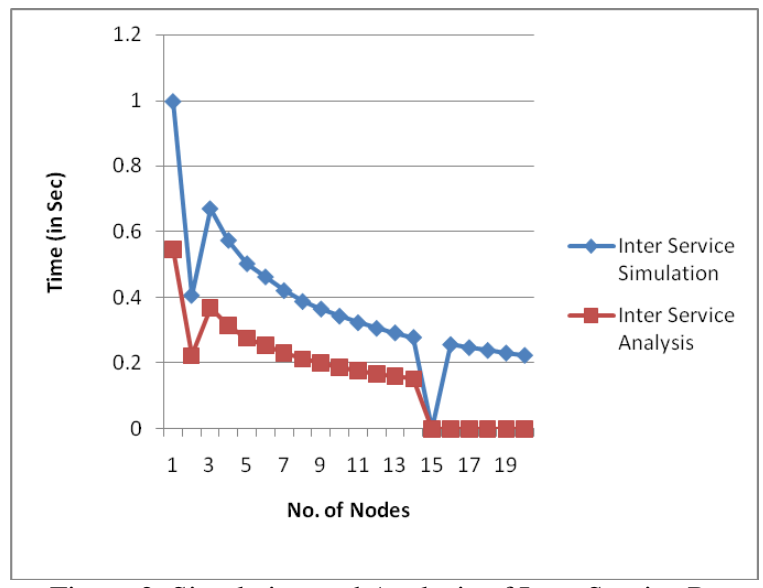

Figure 2. Simulation and Analysis of Inter Service Rate.

Its shows that number of nodes in between 1 and 20. In X Axis and Times in sec provided in Y Axis.

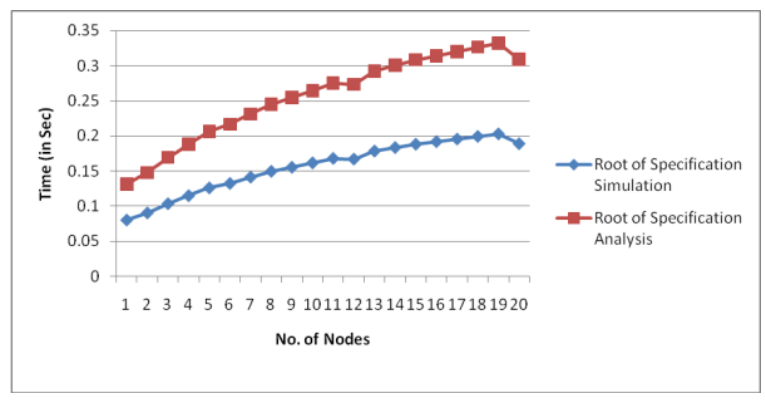

Figure 3.: Simulation and Analysis of Root of Specification.
Its shows that number of nodes in between 1 and 20. In X Axis and Times in sec provided in Y Axis.

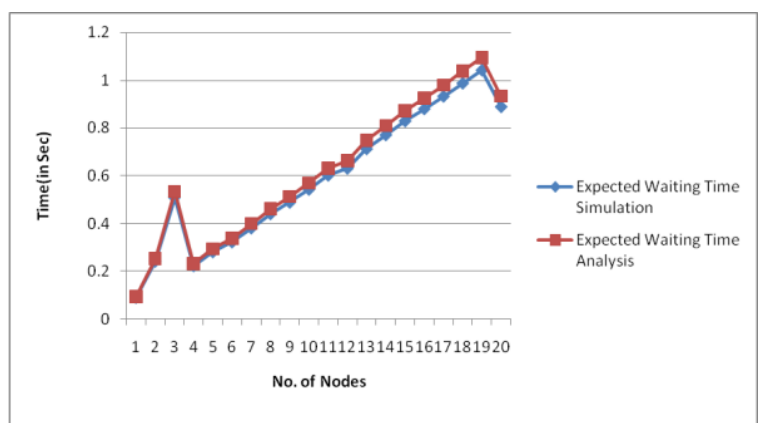

Figure 4. Simulation and Analysis of Expected waiting time.

In this parameter the value of data packets shows the Expected waiting time in between client and server.

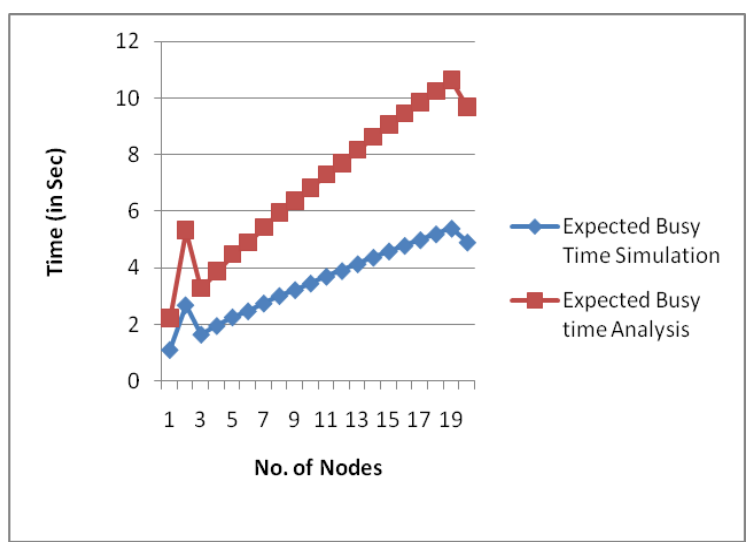

Figure 5. Simulation and Analysis of Expected Busy time.

In this parameter the value of data packets shows the Expected busy period time in between client and server.

By assuming $\lambda$ - Inter arrival time in between client and web server. The values are mentioned in the graph model and $\mu$ inter service time in between client and web server evaluated. After the value is evaluated with $\lambda$ and $\mu$ we have found the root of specification of $0<\mathrm{r}>1$ values. Finally, the Expected waiting time and expected busy period were evaluated by assuming $\lambda, \mu$ and root of specification. It is concluded that the introduction of threshold of 20 nodes which transacts the data packets in between client and web server using switch device, which is idle state to busy state has significant effect with respect to the data arrival and service.

\section{CONCLUSION}

The work concludes that we have presented a model of apache web server using bulk service rule. We have obtained the apache web server performance valid such as average of request and response time and also the expected waiting time and busy period. It has been found that the bulk service rule $\mathrm{M} / \mathrm{M}(1, \mathrm{~b}) / 1$ is better implementation for better performance to fit in the web server with the help of encryption technique. 


\section{REFERENCES}

[1] J.Hu. S.Mungee and D. Schmidt, "Principles for develoing and measuring high-performance web servers over ATM", in proceedings of INFOCOM'98, march/April 1998.

[2] D.A.Menasce and V.A.F.Almeida, capacity planning for webservices, prentice hall,2002.

[3] “Apache web server”, http://www.apache.org.

[4] F.Bause, P. Buchholz, and p. kemper, hierarchically combined queueing petri nets. In proc of $11^{\text {th }}$ intl. conference on analysis and optimization of systems, Discrete event systems, sophie-antipolis france 1994.

[5] Web servers, http://www.knowledgerush.com

[6] Netcraft web server survey, http://www.netcraft.com/survey/archive.html.

[7] J.cao and c.nyberg, "on overload control through queue length for webservers", in $16^{\text {th }}$ Nordic teletraffic seminar, 2002, esboo, finland

[8] J. Dilley, R. Friedrich, T. jin and J.Rolia, web server performance measurement and modeling techniques", performance evaluation. Vol.33, pp. 5-26, 1998.

[9] Performance modeling of an apache web server with bursty arrival traffic,"mikael Anderson, jianhua cao, maria kihl and Christian Nyberg".
[10] J. cao. M. Anderson, C. Nyberg, M.Kihl, Web server performance modeling using an M/G/1/K*PS Queue", in $10^{\text {th }}$ international conference on telecommunications, 2003, papeete, Tahiti.

[11] N. Widell. "Performance of distributed information systems". Department of communication systems, lund institute of technology, tech, rep. 144, 2002, lic. Thesis.

[12] Application of client server networking in wireless technologies by using markov algorithm and queueing petri nets with the security of steganography. V.b.kirubanand, S.palaniammal, CIITpublishers.April 2009.

[13] Performance modeling of an apache web server with dusty arrival traffic. Mikel Anderson, Jianhua cao, Maria Kihl and chistian Nyberg)

[14] F. Bause. "QN+PN=QPN" combining Queuing networks and Petri nets. Technical report no.461.1993.

[15] H. Heffes. "A class of Data Traffic Processes Covariance function charaterizationn and related queuing results" The bell system technical journal, vol. 59, no.6 July-august 1980.

[16] Performance modeling of distributed e-business application using queing petri nets Kounev, S.Buchmann.A. 2003 